\title{
Magnetization Angle and Thickness Dependence of Perpendicular Exchange Anisotropy in $[\mathrm{Pd} / \mathrm{Co}]_{\mathbf{n}} / \mathrm{FeMn}$ Films
}

\author{
S. D. Choi ${ }^{1}$, H. W. Joo ${ }^{1}$, D. K. Yun ${ }^{1}$, M. S. Lee ${ }^{1}$, K. A. Lee ${ }^{1}$, H. S. Lee ${ }^{3}$, \\ S. W. Kim², S. S. Lee ${ }^{2}$, and D. G. Hwang ${ }^{2} *$ \\ ${ }^{1}$ Department of Physics, Dankook University, Cheonan 330-714, Korea \\ ${ }^{2}$ Computer and Electronic Physics, Sangji University, Kangwon 220-702, Korea \\ ${ }^{3}$ Oriental Biomedical Engineering, Sangji University, Kangwon 220-702, Korea
}

(Received 6 March 2006)

\begin{abstract}
The magnetization angle and thickness dependence of magnetic anisotropy in the exchange-biased [Pd/Co] $\times 5 /$ FeMn multilayers with an out-of-plane anisotropy were investigated to determine the origin of perpendicular exchange biasing. As the Co thickness increased to $1.5 \mathrm{~nm}$ in the $[\operatorname{Pd}(0.8 \mathrm{~nm}) / \mathrm{Co}(\mathrm{t})] \times 5 / \mathrm{FeMn}(120 \mathrm{~nm})$ films, the hysteresis loops were converted from square loops at a thin $\mathrm{Co}(<0.4 \mathrm{~nm})$ to complicated round ones at a thick Co. The irregularly asymmetric step (IAS) at the left top of the loop appeared in the loop of the 0.6-nm Co film due to an inhomogeneity in the exchange anisotropy. As the Pd thickness increased to $1.6 \mathrm{~nm}$, the step disappeared, and the perpendicular magnetic anisotropy was maximized in the Co thickness between 0.6 and $0.9 \mathrm{~nm}$. The conversion of the magnetization loop along the magnetization angle coincided with the equation $\boldsymbol{H}_{(e f f)}=\boldsymbol{H}_{o}$ $\cos \theta$. The IAS of the 0.8-nm Pd film disappeared after thermal annealing up to $200{ }^{\circ} \mathrm{C}$ under an external magnetic field.
\end{abstract}

Key words : perpendicular exchange anisotropy, asymmetric step, Co/Pd thickness

\section{Introduction}

Recently, exchange bias effects were observed in $\mathrm{Co} / \mathrm{Pt}$ and $\mathrm{Co} / \mathrm{Pd}$ multilayers with perpendicular anisotropy coupled to an antiferromagnet (AFM) $[1,2]$. The perpendicular magnetic phenomenon can be quite useful for magnetic field sensors and the magnetic recording technique, especially integrated planer sensors, or for MRAM devices. Although perpendicular exchange bias has found applications in spin electronic devices, a magnetic description that satisfactorily explains all observed phenomena has not, as yet, been formulated [3-5].

In this work, the dependence of perpendicular magnetic anisotropy on the magnetization angle and thickness in the $[\mathrm{Pd} / \mathrm{Co}] \mathrm{n} / \mathrm{FeMn}$ film was discussed to explain perpendicular exchange biasing at the interface between Co and FeMn. To eliminate the asymmetric hysteresis loop generated by inhomogeneous exchange coupling at the interface, the Pd thickness dependence and thermal annealing under an external magnetic field were investigated.

*Corresponding author: Tel: +82-33-730-0413,

Fax: +82-33-730-0403, e-mail: dghwang@sangji.ac.kr

\section{Experiments}

$\mathrm{Ta}(2 \mathrm{~nm}) /[\mathrm{Pd} / \mathrm{Co}] \times 5 / \mathrm{FeMn}(120 \mathrm{~nm}) / \mathrm{Ta}(2 \mathrm{~nm})$ multilayers were deposited, using the dc magnetron sputtering system without a magnetic field, under a base pressure of $1.0 \times 10^{-7}$ Torr. The Pd and Co thicknesses changed from 0.8 to $1.6 \mathrm{~nm}$ and from 0.2 to $2.1 \mathrm{~nm}$, respectively. The sample was annealed to $200^{\circ} \mathrm{C}$ under $1.0 \times 10^{-5}$ Torr during an hour. The external magnetic field of $500 \mathrm{Oe}$ was applied during the annealing process. The magnetization curves of the exchange-biased perpendicular films were measured using the extraordinary Hall effect (EHE) and VSM. To investigate the dependence of magnetizing angle on perpendicular magnetic anisotropy, the magnetization curves was obtained under the applied field of 0 , 30, 60 and 90 degree for the out-of-plane direction.

\section{Results and Discussion}

The hysteresis loops of the $[\operatorname{Pd}(0.8 \mathrm{~nm}) / \operatorname{Co}(0.3-1.5$ $\mathrm{nm})] \times 5 / \mathrm{FeMn}(120 \mathrm{~nm})$ multilayers as a function of the magnetizing angle and thickness of Co are shown in Figure 1. The loop of the 0.3-nm Co layer has clear 


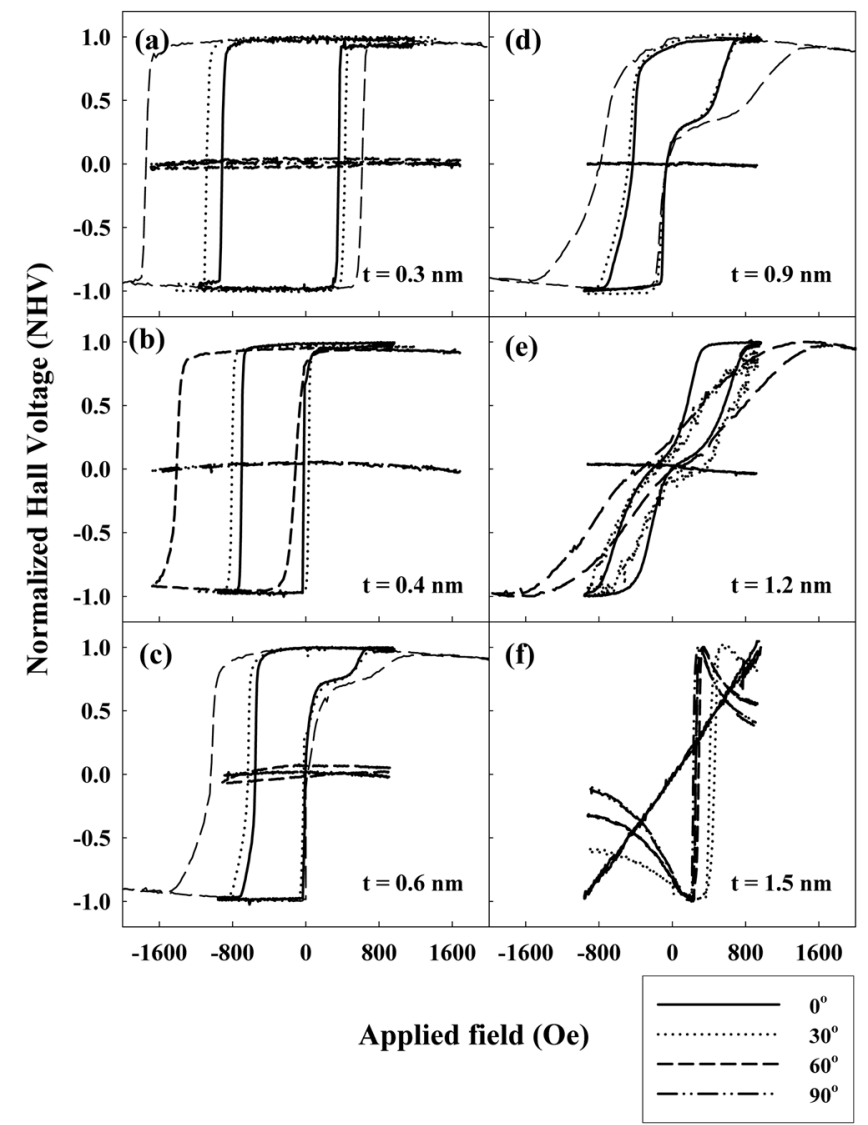

Fig. 1. Normalization Hall voltage of the $\mathrm{Ta}(2 \mathrm{~nm}) /[\operatorname{Pd}(0.8$ $\mathrm{nm}) / \mathrm{Co}(\mathrm{t})] \times 5 / \mathrm{FeMn}(120 \mathrm{~nm}) / \mathrm{Ta}(2 \mathrm{~nm})$ multilayers as a function of Co thickness and the magnetizing angle.

square-type curves due to a strong perpendicular magnetic effective anisotropy, and its exchange biasing field $\left(\boldsymbol{H}_{e x}\right)$ and coercivity $\left(\boldsymbol{H}_{\boldsymbol{c}}\right)$ are $310 \mathrm{Oe}$ and $590 \mathrm{Oe}$, respectively. The perpendicular magnetic effective anisotropy became weak as Co thickness increases, because a decrease of the out-of-plane magnetic effective anisotropy occurs for either very thin or relatively thick Co layers, which drastically reduces perpendicular exchange bias [6]. The IAS at the top of the loop, as shown in figure 1(c) and (d), resulted from the inhomogeneity in the exchange anisotropy. The region of the asymmetric loop was placed at the center of the zero magnetic field. Therefore, the area of the asymmetric loop was proportional to the region uncoupled from the FeMn antiferromagnet at the interface. The 1.5-nm Co sample has in-plane effective anisotropy [6], and perpendicular exchange anisotropy vanished above the 1.2-nm Co sample. The $\boldsymbol{H}_{c}$ 's of the 0.4-nm Co sample were 350, 400, and 650 for the magnetization angle of 0,30 , and 60 degrees, respectively. The $\boldsymbol{H}_{e x}$ 's, on the other hand, were 350,400 , and 700 along the angle. This shows that the conversion of

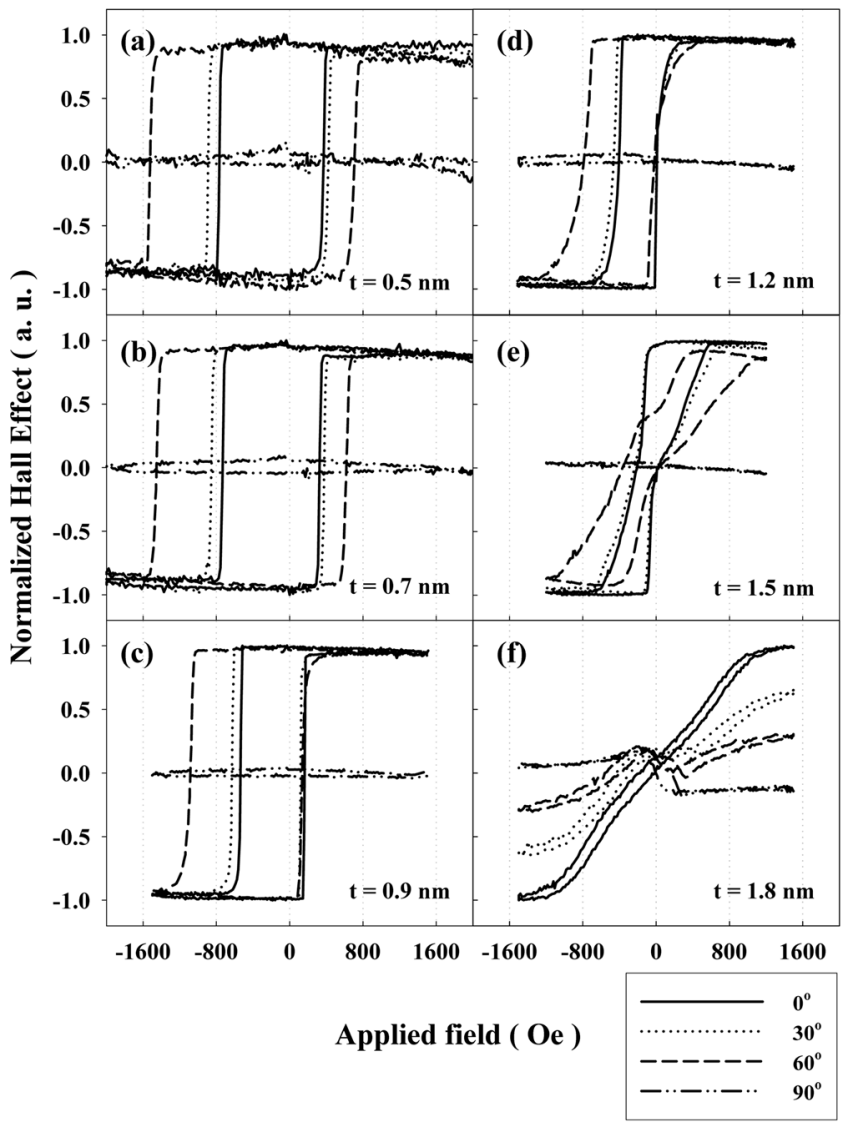

Fig. 2. Normalization Hall voltage of the $\mathrm{Ta}(2 \mathrm{~nm}) /[\operatorname{Pd}(1.6$ $\mathrm{nm}) / \mathrm{Co}(\mathrm{t})] \times 5 / \mathrm{FeMn}(120 \mathrm{~nm}) / \mathrm{Ta}(2 \mathrm{~nm})$ multilayers as a function of Co thickness and the magnetizing angle.

the magnetization loop coincided with the equation $\boldsymbol{H}_{\text {(eff) }}$ $=\boldsymbol{H}_{o} \cos \theta$ as the angle increased [7].

Figure 2 shows the hysteresis loops of the $[\operatorname{Pd}(1.6 \mathrm{~nm}) /$ $\mathrm{Co}(0.3-1.5 \mathrm{~nm})] \times 5 / \mathrm{FeMn}(120 \mathrm{~nm})$ multilayers as a function of Co thickness and the magnetizing angle. The loop of the 0.5- and 0.7-nm Co layer has clear square-type curves, and its $\boldsymbol{H}_{e x}$ and $\boldsymbol{H}_{c}$ are 250 and about $580 \mathrm{Oe}$, respectively. When the Co thickness went beyond $1.2 \mathrm{~nm}$, the loop was converted into a round curve and caused the perpendicular magnetic anisotropy at the $1.8-\mathrm{nm}$ Co multilayers to disappear. In the case of the $1.8-\mathrm{nm} \mathrm{Pd}$ multilayers, the IAS did not appear as the Co thickness increased. This proves that a Pd thickness of below 1.0 $\mathrm{nm}$ can induce inhomogeneous perpendicular exchange anisotropy.

From the hysteresis loops of the 0.8-nm and 1.6-nm Pd films, it can be observed that the loops of Figure 1a $(\mathrm{Pd}=$ $0.8 \mathrm{~nm} ; \mathrm{Co}=0.3 \mathrm{~nm})$ and Figure $1 \mathrm{~b}(\mathrm{Pd}=0.8 \mathrm{~nm} ; \mathrm{Co}=$ $0.4 \mathrm{~nm})$ are similar to those of Figure $2 \mathrm{~b}(\mathrm{Pd}=1.6 \mathrm{~nm}$; $\mathrm{Co}=0.7 \mathrm{~nm})$ and Figure $2 \mathrm{c}(\mathrm{Pd}=1.6 \mathrm{~nm} ; \mathrm{Co}=0.9 \mathrm{~nm})$. This shows that the magnetic properties of the multilayer 


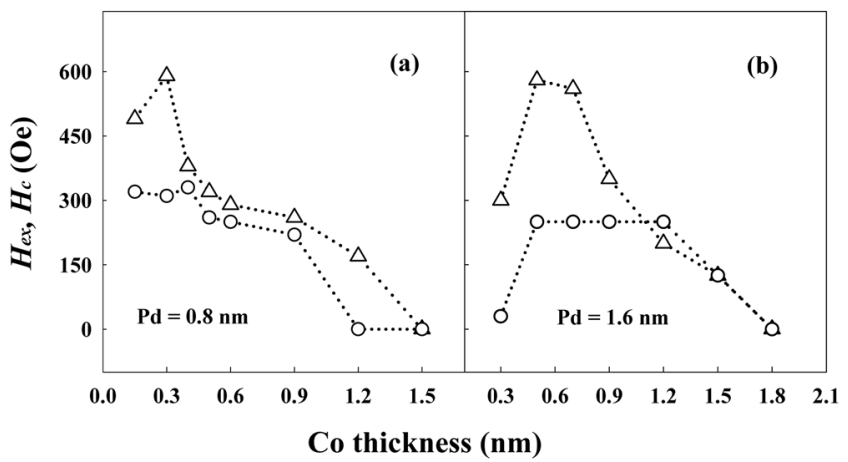

Fig. 3. Changes of $H_{e x}$ (circle) and $H_{c}$ (triangle) as a function of Co thickness in the $\mathrm{Ta}(2 \mathrm{~nm}) /[\operatorname{Pd}(0.8$ and $1.6 \mathrm{~nm}) / \mathrm{Co}(\mathrm{t})] \times 5 /$ $\mathrm{FeMn}(120 \mathrm{~nm}) / \mathrm{Ta}(2 \mathrm{~nm})$ multilayers.

depend on the ratio of the $\mathrm{Co} / \mathrm{Pd}$ thickness rather than on the thickness of each.

Figure 3 shows $\boldsymbol{H}_{e x}$ and $\boldsymbol{H}_{c}$ as functions of the Co thickness of $\mathrm{Pd}=$ (a) $0.8 \mathrm{~nm}$ and (b) $1.6 \mathrm{~nm}$. For $\mathrm{Pd}=0.8$ $\mathrm{nm}, \boldsymbol{H}_{e x}$ and $\boldsymbol{H}_{c}$ were maximized to the Co thickness between 0.3 and $0.4 \mathrm{~nm}$. Above $0.6-\mathrm{nm} \mathrm{Co}$, the valves decreased continuously and became a zero field at 1.2 $\mathrm{nm}$. In the case of $\mathrm{Pd}=1.6 \mathrm{~nm}$, however, the $\boldsymbol{H}_{e x}$ was maximized to 250 Oe at the $0.5 \mathrm{~nm}$ Co thickness, and its value was maintained up to the Co thickness of $1.2 \mathrm{~nm}$. Also, the $\boldsymbol{H}_{\boldsymbol{c}}$ was about 580 Oe at the Co thickness of 0.5$0.7 \mathrm{~nm}$, but was reduced to zero above the Co thickness of $1.8 \mathrm{~nm}$. This shows, therefore, that the perpendicular exchange anisotropy of the $[\mathrm{Pd} / \mathrm{Co}] / \mathrm{FeMn}$ multilayers that have a thick Pd layer is more stable and homogeneous than that of the thin Pd layer. The $\boldsymbol{H}_{e x}$ and $\boldsymbol{H}_{\boldsymbol{c}}$ were at a maximum at $\mathrm{Co}=0.3-0.4 \mathrm{~nm}$ in the case of $\mathrm{Pd}$ $=0.8 \mathrm{~nm}$, and $0.6-0.7 \mathrm{~nm}$ in $\mathrm{Pd}=1.6 \mathrm{~nm}$. This proves that the $\boldsymbol{H}_{e x}$ and $\boldsymbol{H}_{c}$ were optimized when the Co thickness was twice the Pd.

Figure 4 shows the effective magnetic anisotropy $\left(\boldsymbol{K}_{\text {eff }}\right)$

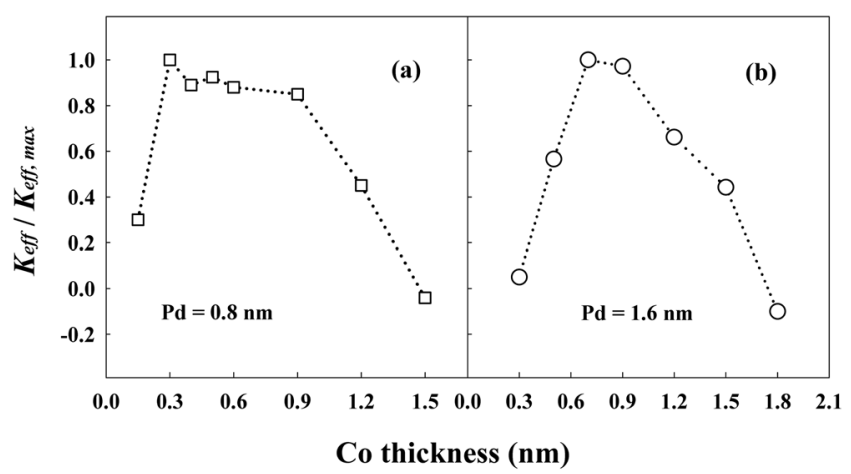

Fig. 4. Changes of $K_{\text {eff }}$ as a function of the Co thickness of the $\mathrm{Ta}(2 \mathrm{~nm}) /[\operatorname{Pd}(0.8$ (a) and $1.6(\mathrm{~b}) \mathrm{nm}) / \mathrm{Co}(\mathrm{t})] \times 5 / \mathrm{FeMn}(120 \mathrm{~nm}) /$ $\mathrm{Ta}(2 \mathrm{~nm})$ multilayers.

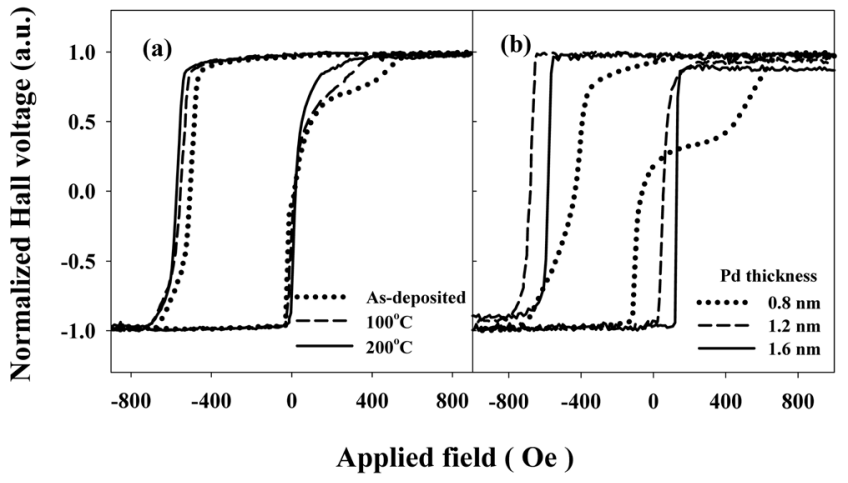

Fig. 5. Normalization Hall voltage of the $\mathrm{Ta}(2 \mathrm{~nm}) /[\operatorname{Pd}(0.8) /$ $\mathrm{Co}(0.5 \mathrm{~nm})] \times 5 / \mathrm{FeMn}(120 \mathrm{~nm}) / \mathrm{Ta}(2 \mathrm{~nm})$ multilayer as a function of the annealing temperature (a) and the $\mathrm{Ta}(2 \mathrm{~nm}) /[\mathrm{Pd}(\mathrm{t}) /$ $\mathrm{Co}(0.9 \mathrm{~nm})] \times 5 / \mathrm{FeMn}(120 \mathrm{~nm}) / \mathrm{Ta}(2 \mathrm{~nm})$ multilayer as a function of the thickness of Pd (b).

as a function of the Co thickness for the Pd thickness of $0.8 \mathrm{~nm}$ and $1.6 \mathrm{~nm} . \boldsymbol{K}_{\text {eff }}$ can be written as $\boldsymbol{K}_{\text {eff }}=\boldsymbol{K}_{V}+(2 n$ $-1) \boldsymbol{K}_{\text {surf }} / n \boldsymbol{t}_{\boldsymbol{C} \boldsymbol{o}}+\boldsymbol{K}_{\text {top }} / n \boldsymbol{t}_{\boldsymbol{C o}}[5,8,9]$, where $n$ is the number of bilayer repeats, $\boldsymbol{K}_{V}$ is the volume anisotropy of the Co layers, $\boldsymbol{K}_{\text {surf }}$ is the perpendicular interfacial anisotropy of the $\mathrm{Co} / \mathrm{Pd}$ interface, and $\boldsymbol{K}_{\text {top }}$ is the interfacial anisotropy of the $\mathrm{Co} / \mathrm{FeMn}$ interface. $\boldsymbol{K}_{\text {eff }}$ was estimated from the difference of the area between the out-of-plane (positive) and in-plane (negative value) hysteresis loops by VSM. For $\mathrm{Pd}=0.8 \mathrm{~nm}$, the $0.3-\mathrm{nm}$ Co layer sample has the highest $\boldsymbol{H}_{c}\left(590\right.$ Oe) and $\boldsymbol{K}_{\text {eff }} / \boldsymbol{K}_{\text {eff, } \text { max }}$ (1.0). The $\boldsymbol{K}_{\text {eff }} /$ $\boldsymbol{K}_{\text {eff, } \max }$ of the 1.5-nm Co sample was a negative value of -0.05 . For Pd $=1.6 \mathrm{~nm}$, however, the $\boldsymbol{K}_{\text {eff }} / \boldsymbol{K}_{\text {eff, } \text { max }}$ of 0.5$\mathrm{nm}$ Co had the maximum value, and its value was maintained up to $0.7 \mathrm{~nm}$ Co thickness. The $\boldsymbol{K}_{\text {eff }} / \boldsymbol{K}_{\text {eff, } \text { max }}$ of the $1.8-\mathrm{nm}$ Co sample was a negative value of -0.1 . This shows that in-plane anisotropy is stronger than the perpendicular one. For the $[\mathrm{Pd} / \mathrm{Co}] / \mathrm{FeMn}$ multilayer structure, the perpendicular magnetic anisotropy became weak for very thin $(<0.5 \mathrm{~nm})$ or very thick Co $(>0.9 \mathrm{~nm})$ [6].

To understand better the IAS of the hysteresis loop in the exchange-biased $[\mathrm{Pd} / \mathrm{Co}]$ films, the $[\operatorname{Pd}(0.8) / \mathrm{Co}(0.5)]$ $\times 5 / \mathrm{FeMn}(12 \mathrm{~nm})$ multilayers were thermally annealed, as shown in Figure 5. In Figure 5(a), the disappearing process of the IAS can be observed with the increase in the annealing temperature. This shows that the sample with an inhomogeneous exchange-coupled interface between $[\mathrm{Pd} / \mathrm{Co}]$ and $\mathrm{FeMn}$ developed a homogeneous interface due to thermal annealing under an external magnetic field. With the increase in Pd thickness, the $\boldsymbol{H}_{\boldsymbol{c}}$ likewise increased from 250 to $350 \mathrm{Oe}$, as shown in Figure 5(b). The IAS also vanished above 1.2-nm Pd. The $\boldsymbol{K}_{\text {eff }} / \boldsymbol{K}_{\text {eff,max }}$ and $\boldsymbol{H}_{e x}$, especially, were maximized to 1.0 
and 330 Oe at 1.2-nm Pd. The $\boldsymbol{K}_{\text {eff }} / \boldsymbol{K}_{\text {eff, max }}$ and $\boldsymbol{H}_{e x}$ of both 0.8 - and 1.6-nm Pd were 0.7 and 250 Oe, respectively.

\section{Conclusion}

Dependence of Co and Pd thicknesses and the magnetizing angle on perpendicular magnetic anisotropy in the exchange-biased $[\mathrm{Pd} / \mathrm{Co}] \times 5 / \mathrm{FeMn}$ multilayers was reported. The magnetization curves were changed from a square loop to a round one with the increase in Co thickness due to weakening a perpendicular anisotropy. The irregulary asymmetric step in the loop of the (Co/Pd- $0.6 / 0.8 \mathrm{~nm})$ film was originated from an inhomogeneity of exchange anisotropy at the interface between $[\mathrm{Pd} / \mathrm{Co}]$ and $\mathrm{FeMn}$. The step disappeared after thermal annealing under an external magnetic field and the increase of Pd thickness.

\section{Acknowledgement}

This work was supported by Korea Research Foundation Grant funded by Korea Government (MOEHRD, Basic Research Promotion Fund) (KRF-2005-D00012).

\section{Rerefences}

[1] W. B. Zeper, H.W. van Kesteren, B. A. J. Jacobs, J. H. M. Spruit, and P. F. Carcia, J. Appl. Phys. 70, 2264 (1991).

[2] M. Kisielewski, A. Maziewski, M. Tekielak, J. Ferru, S. Lemerle, V. Mathod, and C. Chappert, J. Magn. Magn. Mater. 260, 231 (2003).

[3] C. H. Marrows, Phys. Rev. B. 68, 012405 (2005).

[4] F. Garcia, J. Sort, B. Rodmacq, S. Auffret, and B. Dieny, Appl. Phys. Lett. 83, 3537 (2003).

[5] F. Garcia, F. Fettar, S. Auffret, B. Rocmacq, and B. Dieny, J. Appl. Phys. 93, 8397 (2003).

[6] J. Sort, V. Baltz, F. Garcia, B. Rodmacq, and B. Dieny, Phys. Rev. B 71, 054411 (2005).

[7] T. Ambrose, R. L. Sommer, and C. L. Chien, Phsy. Rev. B 56, 83 (1997).

[8] S. van Dijken, M. Besnier, J. Moritz, and J. M. D. Coey, J. Appl. Phys. 97, 10k114 (2005).

[9] H. W. Joo, S. W. Kim, J. H. An, J. H. Choi, M. S. Lee, K. A. Lee, D. G. Hwang, and S. S. Lee, J. of Magnetics 10(1), 33 (2005). 